\title{
Solidification in 4D: from Dendrites to Eutectics
}

Yue Sun ${ }^{1}$, Peter W. Voorhees ${ }^{1}$, A. Shahani ${ }^{2}$, Ahmet Cecen ${ }^{3}$, John W. Gibbs ${ }^{4}$, Surya R. Kalidindi ${ }^{5}$ and X. $\mathrm{Xiao}^{6}$

${ }^{1}$ Department of Materials Science and Engineering, Northwestern University, Evanston, IL

${ }^{2}$ Department of Materials Science and Engineering, University of Michigan, Ann Arbor, MI

${ }^{3}$ School of Computational Science and Engineering, Georgia Institute of Technology, Atlanta, GA

${ }^{4}$ Los Alamos National Laboratory, Los Alamos, NM

${ }^{5}$ George W. Woodruff School of Mechanical Engineering, Georgia Institute of Technology, Atlanta, GA

${ }^{6}$ X-ray Science Division, Advanced Photon Source, Argonne National Laboratory, Lemont, Il

With the advent of high-energy X-ray sources and the development of iterative reconstruction methods, it is now possible to follow solidification processes in three dimensions and as a function of time. The ability to observe and quantify the solidification process in metals on sub-second time scales and micron spatial scales in three dimensions provide fundamentally new insights into this complex phase transformation. We determine the three-dimensional interfacial morphology using a novel X-ray tomographic technique that improves the temporal resolution by more than an order of magnitude compared to conventional techniques.

When group IV semiconductor crystals such as Si and Ge grow from the melt, they form faceted crystals with $\{111\}$ habit planes. The $\{111\}$ is the densely packed cleavage plane in the diamond cubic structure; it is now well established that $\{111\}$ is both the low mobility and low energy plane. During crystal growth, faceted interfaces may be perturbed by defects, leading to a rich variety of polycrystalline growth forms that allow the interfaces to propagate. One such defect is the coherent $\Sigma_{3}\{111\}$ twin boundary, which is widely known to catalyze crystal growth. Controversial mechanisms have been proposed for this process, most of which lack experimental verification. The real-time interfacial dynamics of polycrystalline $\mathrm{Si}$ particles growing from an $\mathrm{Al}-\mathrm{Si}-\mathrm{Cu}$ liquid was examined. Our novel analysis of the time evolution of the interfacial normals allows us to quantify unambiguously the habit plane and grain boundary orientations during growth. This, when combined with direct measurements of the interfacial morphology provide the first confirmation of twin-mediated growth, proposed over 50 years ago [2].

The eutectic morphologies that may arise during solidification can be classified as either regular or irregular. Regularity refers to the periodic arrangement of lamellae, and is typical of non-faceted systems. The situation is more complex when irregular eutectics are considered, in which one of the phases is faceted (for example, $\mathrm{Si}$ and $\mathrm{Ge}$ ) and the other non-faceted (for example, $\mathrm{Al}$ and $\mathrm{Ag}$ ). Using time resolved X-ray tomography, we identified the growth mode of irregular eutectics [3], see Figure 1. Our results show that the eutectic growth process can be markedly different from that seen in previously used model systems and theories based on the ex situ analysis of microstructure.

Like many other microstructures present in the nature, dendrites are complex structures. They have irregular shapes, and are often highly interconnected. Moreover, as most real problems demand threedimensional or even four-dimensional (including time) considerations, it makes it practically difficult 
to visualize the data, and even more so to analyze it. As a result, two-dimensional sections of the data are usually made in order to obtain salient attributes of the three-dimensional dataset, such as the secondary and tertiary arm spacings of the dendrite. As the system size grows larger, the dataset may become unwieldy to handle as a whole, and hence data processing and interpretation must be done in a piece by piece manner. This adds significantly to the challenges in arriving at meaningful global (bulk) descriptors of the microstructure that are also of high value in correlating to its performance characteristics. We extend the existing framework of two-point spatial correlations to allow for the quantification, analysis, interpretation and visualization of microstructure. We examine coarsening of dendritic structures measured by time-resolved X-ray computed tomography. Specifically, extensions were made to facilitate (i) the incorporation of nonconventional local attributes such as the solid-liquid interface, interface curvature, and interface velocity in the description of the local state, and (ii) the efficient computation of bulk spatial correlations when the local attributes are sparsely defined only at special locations in the three-dimensional volume (e.g., solid-liquid interfaces). Algorithmic enhancements needed to carry out these computations on the large datasets produced in the experiments will also be discussed. The results demonstrate the remarkable ability of these new protocols in automated (unbiased) capture of the four-fold symmetry of the dendritic microstructure, and in providing quantitative and reliable estimates of the characteristic lengths associated with the dendritic microstructure (including the secondary and tertiary dendrite arm spacings, secondary dendrite arm diameter, and the solute diffusion length).

\section{References:}

[1] A.J. Shahani et al, Voorhees, Scientific Reports, 6 (2016), p. 28651.

[2] Shahani, A. J.; Xiao, X.; and Voorhees, P. W., Nature Comm. 7 (2016), p. 12953.

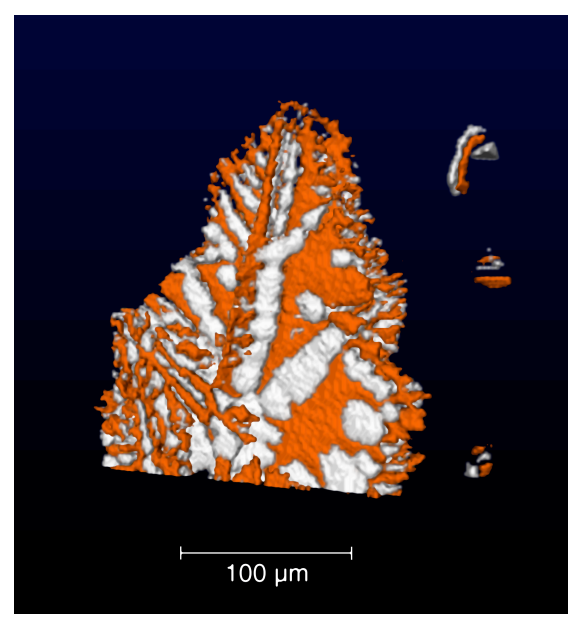

Figure 1. A snapshot of an eutectic colony in the Al-Ge system. The faceted Ge phase is orange and the Al phase is white. Evident is the growth the Al phase along the faceted $\{111\}$ planes of the Ge. The eutectic colony is growing upwards. 